\title{
Optimal Design of Single Phase Permanent Magnet Brushless DC Motor Using Particle Swarm Optimisation
}

\section{Structured Abstract:}

Purpose - The purpose of this paper is to perform an optimal design of single-phase permanent magnet brushless DC motor (SPBLDCM) using efficiency of the motor as objective function. In the design procedure performed on SPBLDCM, particle swarm optimisation (PSO) as an optimisation tool is used.

Design/methodology/approach - The created computer programme for optimal design of electrical machines is based on the particle swarm optimisation. According to the design characteristics of SPBLDCM, some of the motor parameters are chosen to be constant and others variable. A comparative analysis of both motor models based on the value of the objective function, as well as the values of the optimisation parameters, is performed.

Findings - From the comparative data analysis of both motor models, it can be concluded that the main objective of the optimisation is realised, and it is achieved by an improvement of the efficiency of the motor.

Originality/value - An optimisation technique based on PSO has been developed and applied to the design of SPBLDCM. According to the results it can be concluded that the PSO is a very suitable tool for design optimisation of SPBLDCM and electromagnetic devices in general. The quality of the PSO model has been proved through the data analysis of the prototype and optimised solution. At the end, the quality of the PSO solution has bee again proved by comparative analysis of the two motor models using FEM as a performance analysis tool.

Keywords: design optimisation; brushless motors; permanent magnet machine; FEM.

\section{Article Classification: Research paper}

For internal production use only

\section{Running Heads:}

\section{Introduction}

Efficiency improvement of electrical machines has become of wide interest to researchers and designers, due to the increase of electricity demand and price, as well as the stiff competition on the market. During the years different optimisation algorithms have been implemented in the design procedures for a variety of electrical machines. When optimising an electric motor, there are multiple choices of the objective function available. The objective function is the specific property of the machine to be optimised, for example efficiency, torque, volume or cost. The work in this paper will be focused on the optimisation of a motor design using efficiency as an objective function.

In general the optimal design of electrical machines is a constrained maximisation/minimisation problem with a large number of optimisation parameters and variety of constraints. This makes it a difficult problem to solve for the deterministic methods, but on the other hand quite an easy task for the stochastic methods, especially for Particle Swarm Optimisation (PSO) method. In this work an optimal design of a single-phase permanent magnet brushless DC motor using efficiency as objective function is performed. In the design procedure performed on the brushless PM motor, particle swarm optimisation, as an optimisation tool is used.

\section{Description of Particle Swarm Optimisation}

The initial PSO algorithm (Kennedy et al., 2001), ( Kennedy and Eberhart,1995), (Eberhart and Kennedy, 1995) was inspired by the social behaviour of biological organisms, and the ability of groups of some species of animals to work as a whole in locating desirable positions in a given area. Such behaviour can be associated with the birds flocking to a food source. This behaviour is very much in relation to the optimisation search for a solution to a non-linear function in a real number search space.

In the PSO search the particles move through the search area using a combination of an attraction to their best solution they have independently found, as well as to the attraction to the best solution that any particle in the neighbourhood has found. The algorithm, which is based on a metaphor of social interaction, searches the space by adjusting the trajectories of moving points in a multidimensional space. The main advantages of PSO algorithm are summarised as simple concept, easy implementation, robustness to control parameters, and computational efficiency when compared with deterministic methods and other stochastic optimisation techniques. Unlike the other heuristic techniques, PSO has a flexible and well balanced mechanism to enhance and adapt both to the global and local exploration abilities. The PSO concept adjusts, at each time step, the velocity of each particle while moving toward its personal best and global best locations. The acceleration is weighted by a random term, with separate random numbers being generated for acceleration toward personal best and global best.

The advantages of PSO over other traditional optimisation techniques can be summarized as follows:

a. PSO is a population-based search algorithm (i.e., PSO has implicit parallelism). This property ensures PSO search not to get trapped in a local extreme.

b. PSO uses payoff (performance index or objective function) information to guide the search in the problem space. Therefore, PSO can easily deal with non differentiable objective functions. Additionally, this property relieves PSO of assumptions and approximations, which are often required by traditional optimization methods.

c. PSO uses probabilistic transition rules and not deterministic rules. Hence, PSO is a kind of stochastic optimization algorithm that can search a complicated and uncertain area. This makes PSO more flexible and robust than conventional methods. 
d. Unlike GA and other heuristic algorithms, PSO has the flexibility to control the balance between the global and local exploration of the search space. This unique feature of PSO overcomes the premature convergence problem and enhances the search capability.

e. Unlike the traditional methods, the solution quality of the proposed approach does not rely on the initial population. Starting anywhere in the search space, the algorithm ensures the convergence to the optimal solution.

A block diagram of the PSO main steps is presented in Fig. 1.

\section{START}

1

\section{Specify the parameters of PSO}

$\checkmark$

Generate initial population

Iter. $=1$

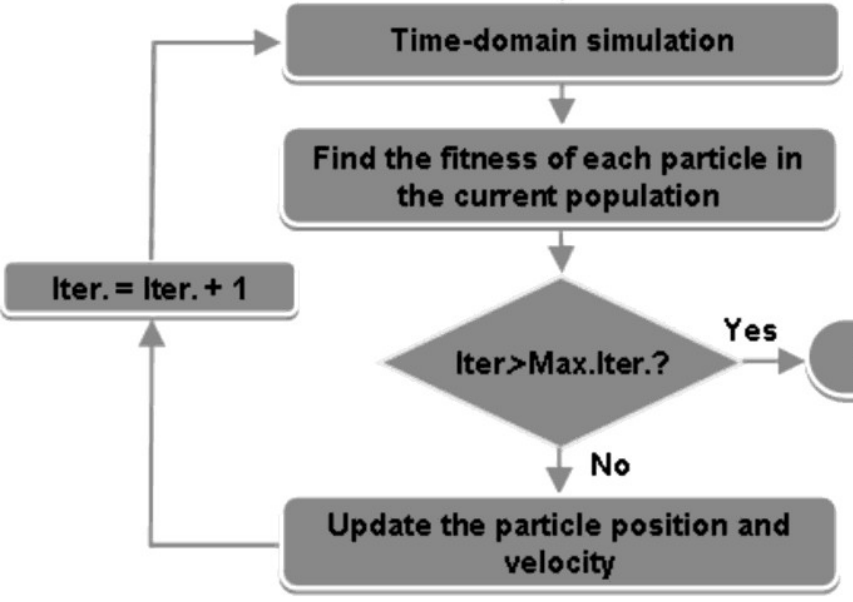

Figure 1. PSO main steps

In the following text the basic elements of PSO technique are briefly stated and defined as follows:

$>$ Particle $X(t)$ : It is a candidate solution represented by an $m$-dimensional real-valued vector, where $m$ is the number of optimised parameters. At time $t$ the $j$-th particle can be described as $X_{j}(t)=\left[x_{j, 1}(t), x_{j, 2}(t), \ldots x_{j, m}(t)\right]$; here ' $x$ ' are the optimised parameters, and $\mathrm{x}_{\mathrm{j}, \mathrm{k}}(\mathrm{t})$ is the position of the particle with respect to the $k$-th dimension (i.e., the value of the $k$-th optimised parameter in the $j$-th candidate solution).

$>$ Population pop $(t)$ : It is a set of $n$ particles at time $t$ (i.e., $\left.p o p(t)=\left[X_{1}(t), X_{2}(t), \ldots X_{n}(t)\right]^{T}\right)$.

$>$ Swarm: It is an apparently disorganised population of moving particles that tend to cluster together while each particle seems to be moving in a random direction.

$>$ Particle Velocity $V(t)$ : It is the velocity of the moving particles represented by an m-dimensional real valued vector.

$>$ Weighting Function $w(t)$ : It is a control parameter that is used to control the impact of the previous velocities on the current velocity. Hence, it influences the trade-off between the global and local exploration abilities of the particles.

$>$ Individual Best $\mathrm{X}^{*}(t)$ : As a particle moves through the search space, it compares its fitness value at the current position to the best fitness value it has ever attained at any time up to the current time. The best position that is associated with the best fitness encountered so far is called the individual best $X^{*}(t)$.

$>$ Global Best $\mathrm{X}^{* *}(t)$ : It is the best position among all of the individual best positions achieved so far.

$>$ Stopping Criteria: These are the conditions under which the search process will terminate. In this study, the search will terminate if the number of iterations reaches the maximum predefined number.

The prescribed values of the PSO parameters are presented in Table 1.

Table 1. The values of the PSO Parameters

\begin{tabular}{|l|c|}
\hline PSO Parameters & Value \\
\hline $\begin{array}{l}\text { PSO parameters } \\
\text { (variables) }\end{array}$ & 6 \\
\hline Population size & 30 \\
\hline Number of iterations & 100 \\
\hline
\end{tabular}




\section{Implementation of PSO in Optimal Design of SPBLDCM}

The design optimisation is performed on a previously defined topology of single phase brushless DC motor [4] with rated voltage, $300 \mathrm{~V}$, and speed, $1500 \mathrm{rpm}$. The single phase brushless DC motor is a four pole motor with concentrated windings mounted on the asymmetrical stator poles and has 4 permanent magnets with $B_{r}=1.13 \mathrm{~T}$ mounted on the rotor. $A$ partial $2 \mathrm{D}$ crosssection presentation of the prototype motor is shown in Fig. 2. It should be mentioned that the motor has an asymmetrical air gap, which is made by modifying the small stator poles, and the rotor PMs (Ahmed and Lefley, 2009).

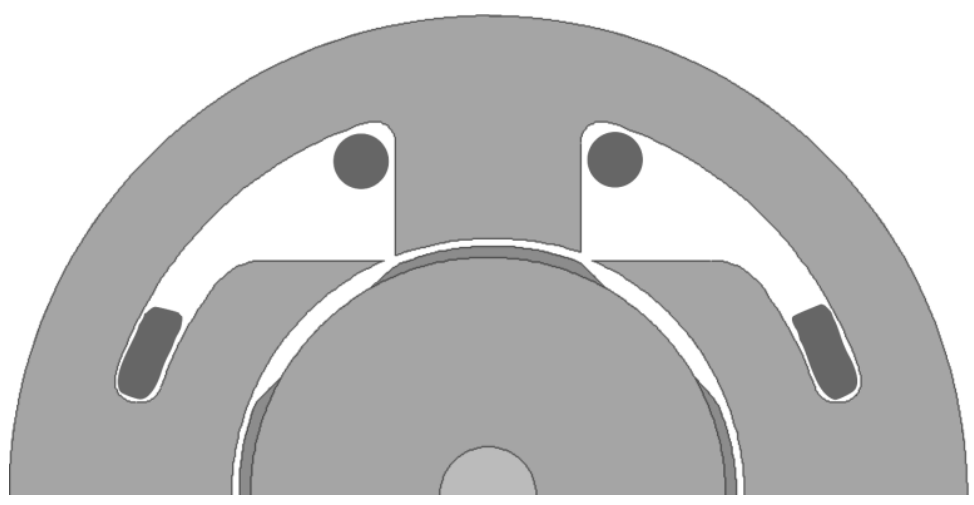

Figure 2. Partial presentation of the SPBLDCM prototype

The efficiency of the motor is selected as an objective function of the optimisation, since the energy saving is especially important for the SPBLDCM. According to the design characteristics of the designed by hand prototype SPBLDCM, some of the parameters are chosen to be constant and some variable, such as: outside radius of the rotor core $\boldsymbol{R}_{\text {ro, }}$ permanent magnet radial thickness $\boldsymbol{I}_{\boldsymbol{m}}$, air-gap $\boldsymbol{g}$, slot opening $\boldsymbol{b}_{\mathbf{s o}}$, axial active length of the motor $\boldsymbol{L}$, and single wire radius $\boldsymbol{r}_{\boldsymbol{c} u}$. Some of these parameters are presented in Figure 3. The objective function for the optimisation is presented by equation (1). The optimisation parameters are selected based on their direct influence on the motor design, as well as the optimisation process, the value of the objective function and on authors' previous experience.

efficiency $=\eta=\frac{T \cdot \omega_{m}}{T \cdot \omega_{m}+P_{C u}+P_{F e}+P_{s}}$

where: $T$-rated torque, $\omega_{m}$-rated speed, $P_{C u}$-ohmic power losses, $P_{F e}$-stator iron core power losses and $P_{s}$-other constant losses. The optimal design process of the single phase brushless DC motor is a maximisation problem of the objective function, where the torque is one of the predefined constraints and is kept constant during the optimization. In this optimisation process the calculation of the losses is done for the rated speed and frequency and for slightly changed stator current. The change of the stator current is due to the change of the magnetic circuit (motor structure) and stator winding parameters at constant torque load. The mathematical model of the motor developed for the optimisation process is validated by comparing the measured values with the calculated ones. They show good agreement and therefore it can be concluded that the optimal PSO solution will be also in good agreement with the physical model of the motor that will be developed in near future.

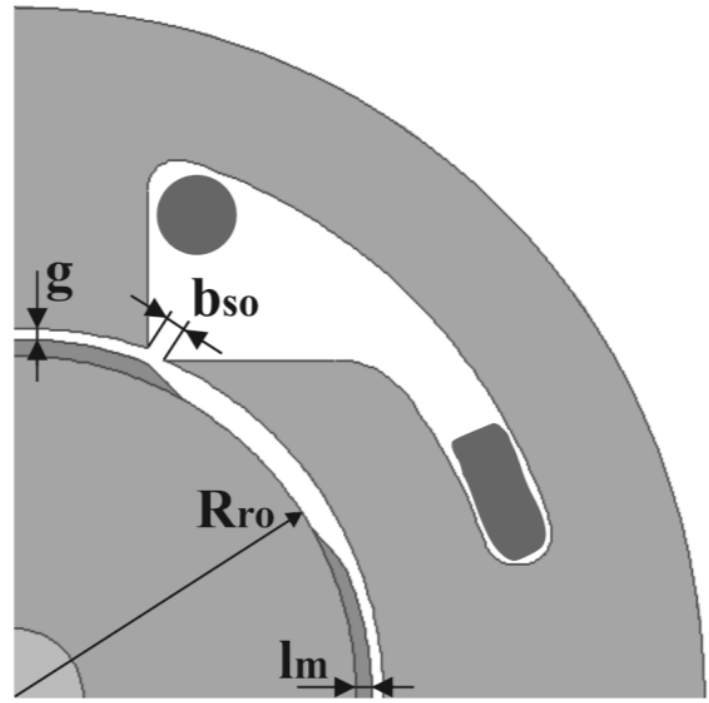

Figure 3. SPBLDCM optimisation parameter presentation 
Some of the design constraints used in the optimal design of the single phase brushless DC motor are geometrical, and the other constraints concern the motor performance and material characteristics. The choice of these constraints has been carefully selected to reduce the number of independent design variables. This is obtained by a steady-state analysis of the motor, which allows the main electrical, magnetic and mechanical quantities, including the set of motor specifications, to be expressed as functions of its dimensions and working conditions. Some of them are presented in Table 2.

Table 2. Optimisation Constraints

\begin{tabular}{|l|c|c|}
\hline Description & Parameters & Value \\
\hline Torque & $T(\mathrm{Nm})$ & 6.366 \\
\hline Input voltage & $U_{p h}(\mathrm{~V})$ & 300 \\
\hline Number of phases & $N_{p h}$ & 1 \\
\hline PM residual flux density & $B_{r}(\mathrm{~T})$ & 1.13 \\
\hline Number of PM & $N_{m}$ & 4 \\
\hline Number of stator poles & $\mathrm{Z}$ & 4 \\
\hline Stator back iron flux density & $B_{m b i}(\mathrm{~T})$ & $\leq 1.0$ \\
\hline Stator teeth flux density & $B_{m s t}(\mathrm{~T})$ & $\leq 1.0$ \\
\hline Stator and rotor steel core loss & $G_{F e}(\mathrm{~W} / \mathrm{kg})$ & 6 \\
\hline Stator conductor resistivity & $\rho_{\mathrm{Cu}}(\Omega \mathrm{m})$ & $1.7510^{-8}$ \\
\hline Stator steel mass density & $\rho_{\mathrm{st}}\left(\mathrm{kg} / \mathrm{m}^{3}\right)$ & 7300 \\
\hline Permanent magnets mass density & $\rho_{P M}\left(\mathrm{~kg} / \mathrm{m}^{3}\right)$ & 7400 \\
\hline Rotor steel mass density & $\rho_{\mathrm{rot}}\left(\mathrm{kg} / \mathrm{m}^{3}\right)$ & 7850 \\
\hline Copper mass density & $\rho_{\mathrm{Cu}}\left(\mathrm{kg} / \mathrm{m}^{3}\right)$ & 8930 \\
\hline
\end{tabular}

Table 3. PSO Parameter Optimisation Bounds

\begin{tabular}{|l|c|c|c|}
\hline Parameters & Lower bound & Upper bound & Basic model \\
\hline$R_{\text {ro }}(\mathrm{m})$ & 0.0342 & 0.0418 & 0.0380 \\
\hline$I_{m}(\mathrm{~m})$ & 0.0018 & 0.0022 & 0.0020 \\
\hline$g(\mathrm{~m})$ & 0.0009 & 0.0011 & 0.0010 \\
\hline$b_{\text {so }}(\mathrm{m})$ & 0.0020 & 0.0030 & 0.0023 \\
\hline$L(\mathrm{~m})$ & 0.0972 & 0.1188 & 0.1080 \\
\hline$r_{\text {cu }}(\mathrm{m})$ & 0.0003 & 0.0005 & 0.0004 \\
\hline
\end{tabular}

The stopping rule of the programme PS-ODEM (Particle Swarm for Optimal Design of Electrical Machines) is selected to be the number of iterations. The lower and upper bound, and the optimisation parameters values of the prototype model, are presented in Table 3.

Table 4. Particle Swarm Optimisation Results

\begin{tabular}{|l|c|c|c|}
\hline Variables & Units & Basic model & PSO Solution \\
\hline$R_{r o}$ & $(\mathrm{~m})$ & 0.038 & 0.0418 \\
\hline$I_{m}$ & $(\mathrm{~m})$ & 0.002 & 0.00194 \\
\hline$g$ & $(\mathrm{~m})$ & 0.001 & 0.0009 \\
\hline$b_{s o}$ & $(\mathrm{~m})$ & 0.0023 & 0.003 \\
\hline$L$ & $(\mathrm{~m})$ & 0.108 & 0.0972 \\
\hline$r_{c u}$ & $(\mathrm{~m})$ & 0.0004 & 0.0005 \\
\hline Efficiency & $(/)$ & 0.8823 & 0.9049 \\
\hline
\end{tabular}




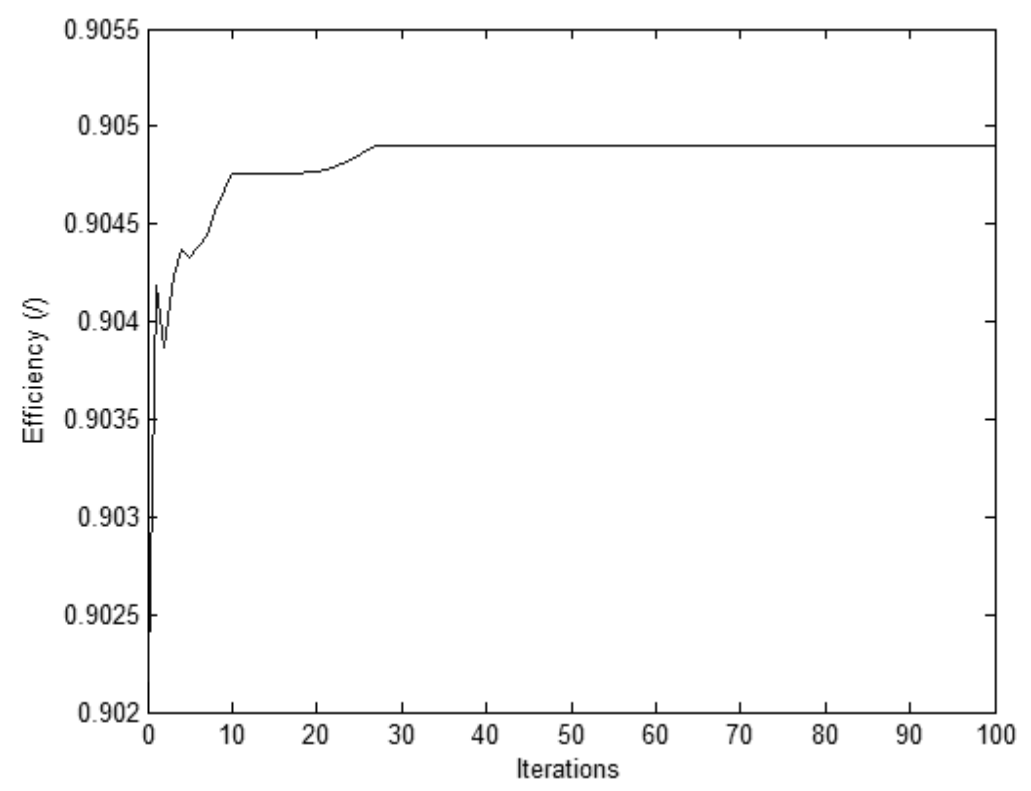

Figure 4. Efficiency change during PSO search

The comparative optimisation parameters data of the basic and optimised model are presented in Table 4. The convergence of the efficiency of the motor as an objective function during the particle swarm optimisation search for 100 iterations is shown in Fig. 4.

Table 5. Initial Motor and PSO Solution Data Comparison

\begin{tabular}{|l|l|c|c|}
\hline Parameters & Description & Initial Motor & PSO Solution \\
\hline$N(/)$ & total number of turns of stator winding & 648 & 622 \\
\hline$X_{n}(\mathrm{~m})$ & $\begin{array}{l}\text { gap between the pole shoe and the } \\
\text { stator inner radius }\end{array}$ & 0.00757 & 0.01023 \\
\hline$X_{c}(\mathrm{~m})$ & $\begin{array}{l}\text { stator pole shoe thickness stator back } \\
\text { iron thickness }\end{array}$ & 0.014 & 0.01527 \\
\hline$X_{p}(\mathrm{~m})$ & stator pole width & 0.0280 & 0.0305 \\
\hline$R_{s o}(\mathrm{~m})$ & stator outside radius & 0.0766 & 0.0854 \\
\hline$I_{p h}(\mathrm{~A})$ & phase current & 3.28 & 3.50 \\
\hline$R_{p h}(\mathrm{ohm})$ & phase resistance & 6.987 & 5.045 \\
\hline$P_{C u}(\mathrm{~W})$ & ohmic losses & 91.38 & 61.86 \\
\hline$P_{F e}(\mathrm{~W})$ & iron losses & 32.06 & 33.18 \\
\hline$\eta(/)$ & efficiency & 0.8823 & 0.9049 \\
\hline
\end{tabular}

\section{PSO Optimal Design Results Of SPBLDCM}

In order to be able to compare the two solutions some specific parameters of the prototype and of the PSO optimal solution are shown in Table 5.

The new optimised values of the parameters and characteristics show improvement of the single phase brushless DC motor model in relation to the prototype. It is evident that the PSO optimized solution has a better efficiency, which is due to the increased cross-section of the stator winding wire and hence a decrease of the resistance of the stator winding. The decrease of the stator winding resistance results in a decrease of the ohmic losses, and hence an improvement in the efficiency of the PSO solution. A structural comparison of the two models is presented in Fig. 5. The top model is the initial model and the bottom model is the PSO solution. From the figure and the data presented in Table 4 and Table 5 it can be noticed that the dimensions of the magnetic circuit of the PSO motor solution changed in relation to the initial motor model. In order to check how this will influence the overall performance of the motor a proper performance analysis of both motor models has to be done.

In order to be able to perform a more detailed comparative analysis of the two motor models a finite element method approach was adopted. FEM analysis is regarded as a very sophisticated and frequently used tool for motor analysis especially for motors that are in the developing stage. In the near future it is planned the optimized motor model to be build and after that to perform some experimental investigation on it and compare the results with the prototype measured ones. This will be of great importance in the validation of the optimization process, as well as the optimized motor model. 


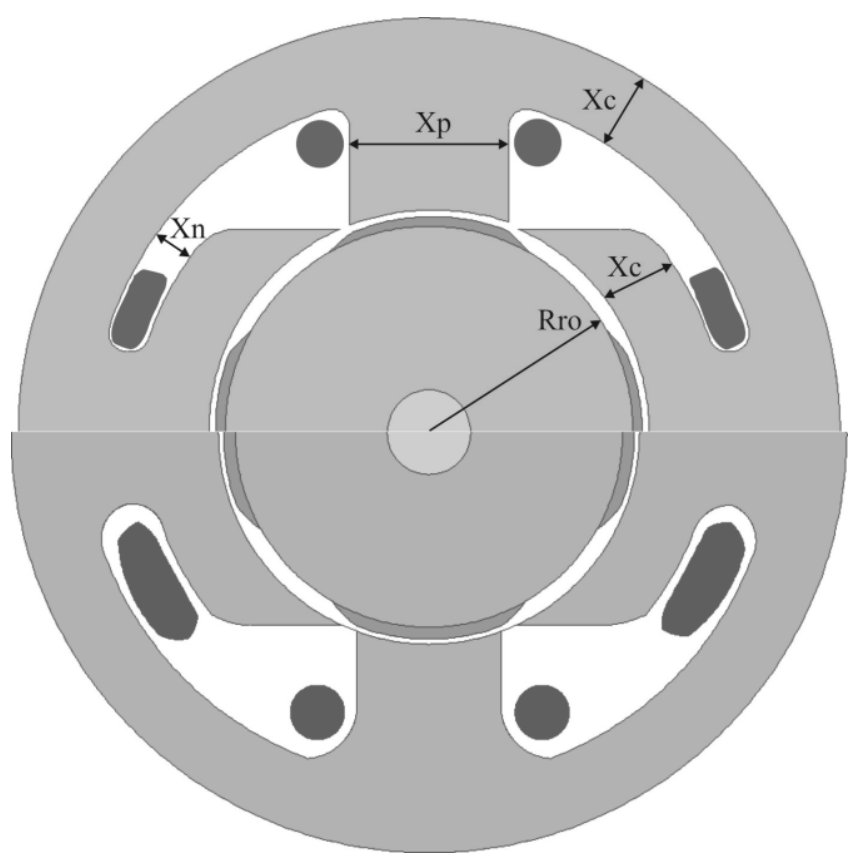

Fig. 5. Presentation of the two SPBLDCM models

\section{SPBLDCM FEM Modelling}

Finite element method (FEM) is one of the tools that has been used successfully for many years for performance analysis of electrical machines. Therefore, this method is also used for performance analysis of the two motor models under investigation in this work. The software used for this purpose is called Finite Element Method Magnetics-FEMM (Meeker, 2010). In order to be able to analyse the two models of the SPBLDC motor accurately, a calculation of the magnetic field has to be performed for the two motor topologies (lepure et al., 2008), (Lefley et al., 2010). The 2D analysis is very suitable for this type of geometry and has a lot of advantages over the 3D calculation, such as lower memory storage and reduced computation time. After the motor has been properly modeled, a 2D FEM mesh can be generated. The mesh for the two motor models consisted of 91125 nodes and 181478 elements. A 2D mesh representation for the single phase brushless DC motor models with fewer finite elements for a better view is presented in Fig. 6. An enlarged view of the finite elements in the air gap is presented in Fig. 7.

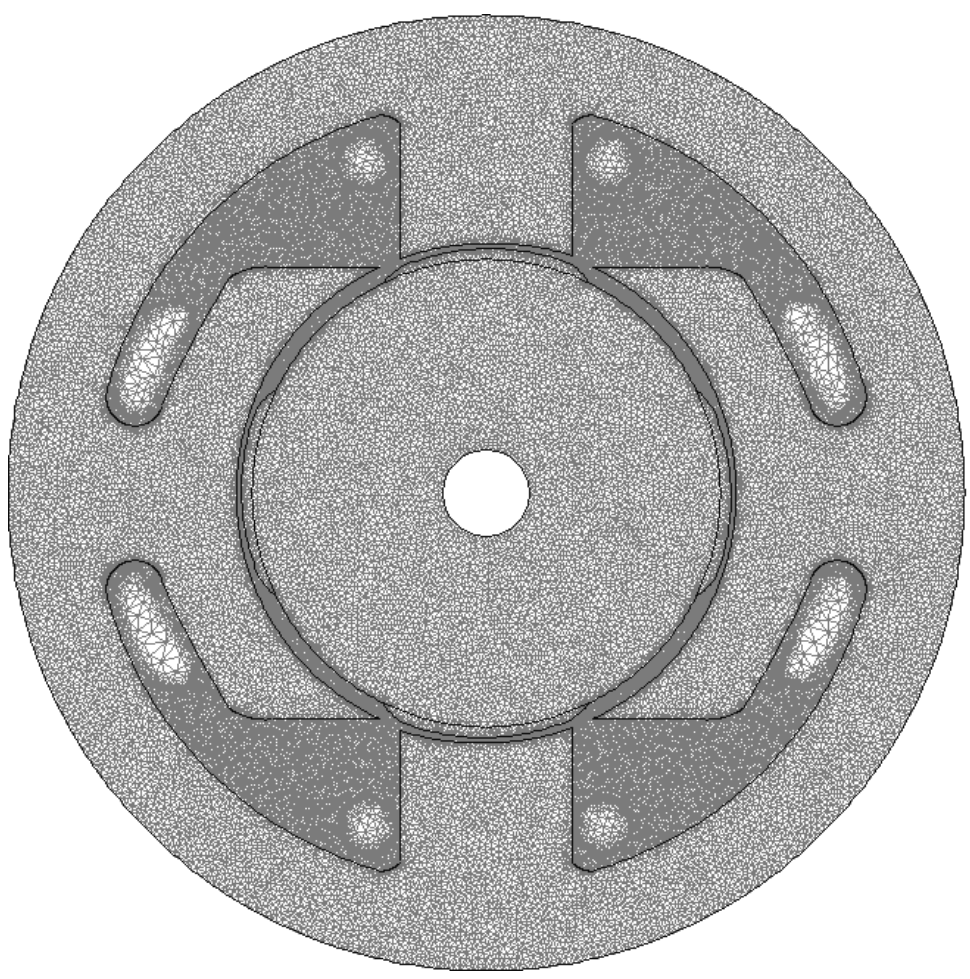

Fig. 6. 2D mesh representation of the SPBLDCM 


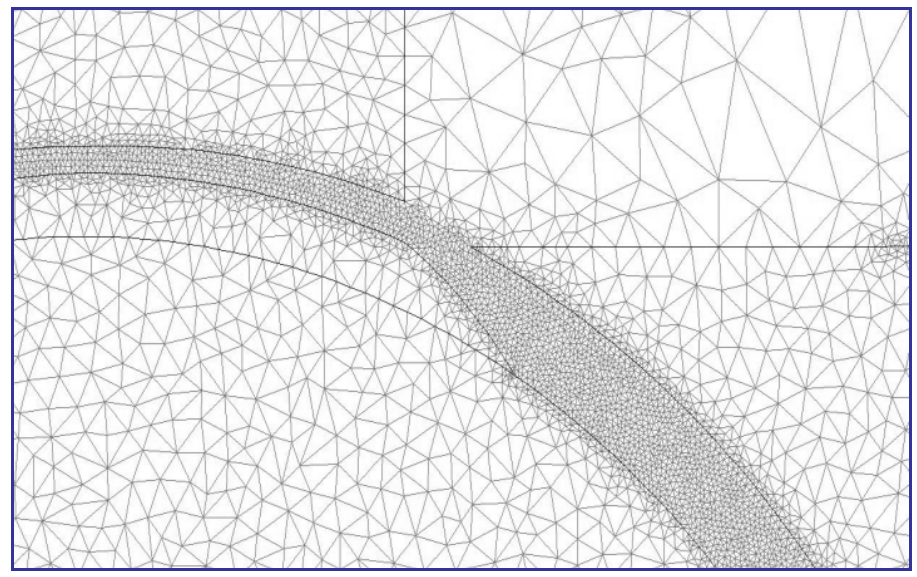

Fig. 7. 2D partial mesh representation of the SPBLDCM in the air gap

\section{FEM Magnetic Field Analysis}

After modelling the single phase brushless DC motor and with adequate mesh size refinement, especially in the air gap, a magnetic field calculation was performed for different load currents and for different rotor displacements. As an example, the magnetic field distribution within the motor at no load and rated load, for both the initial motor model and the PSO solution is presented in Fig 8, Fig. 9, Fig. 10 and Fig. 11, respectively.
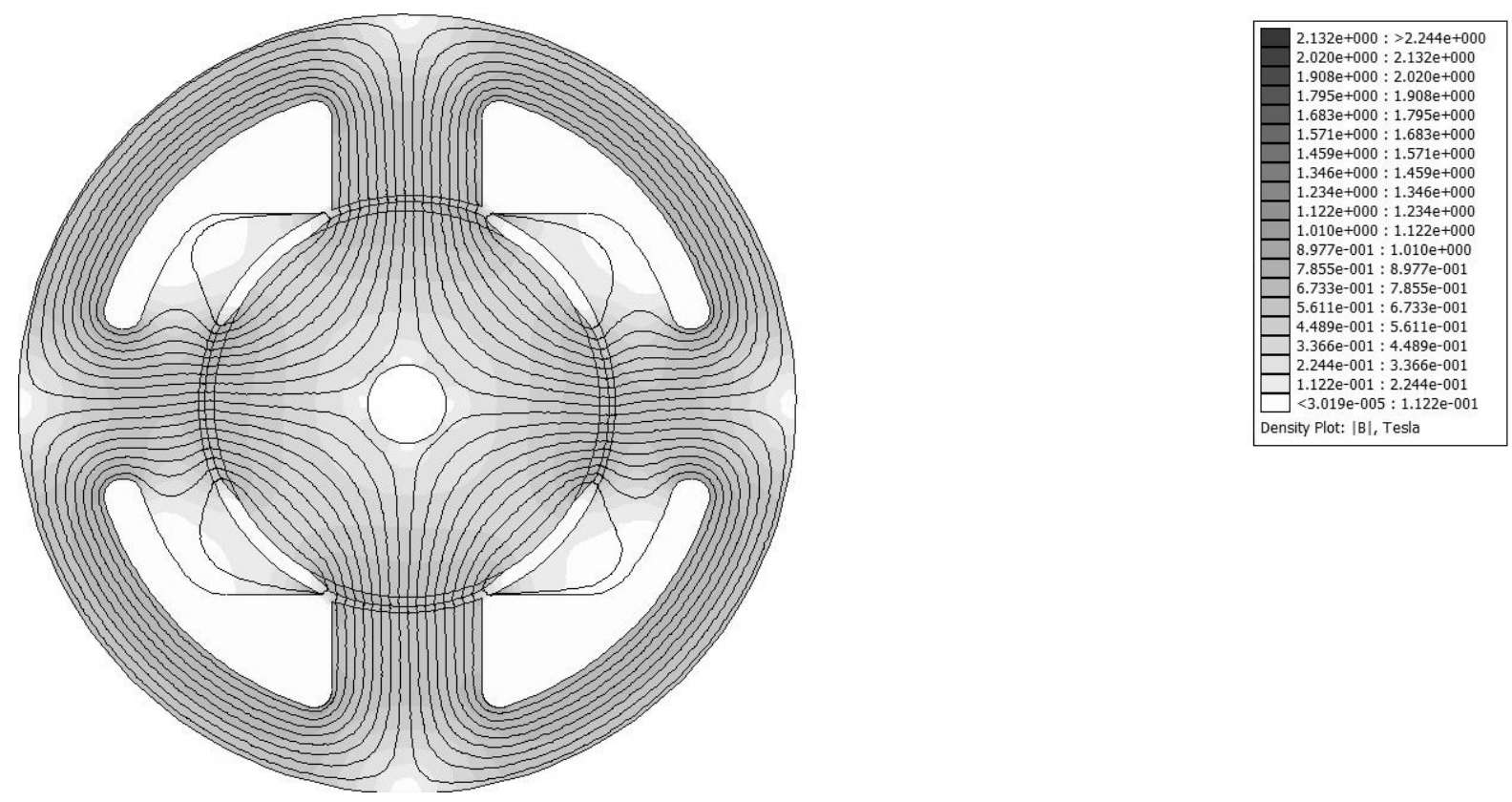

Fig. 8. Magnetic field distribution at no load for the initial model when the axes of the stator and rotor poles are aligned 

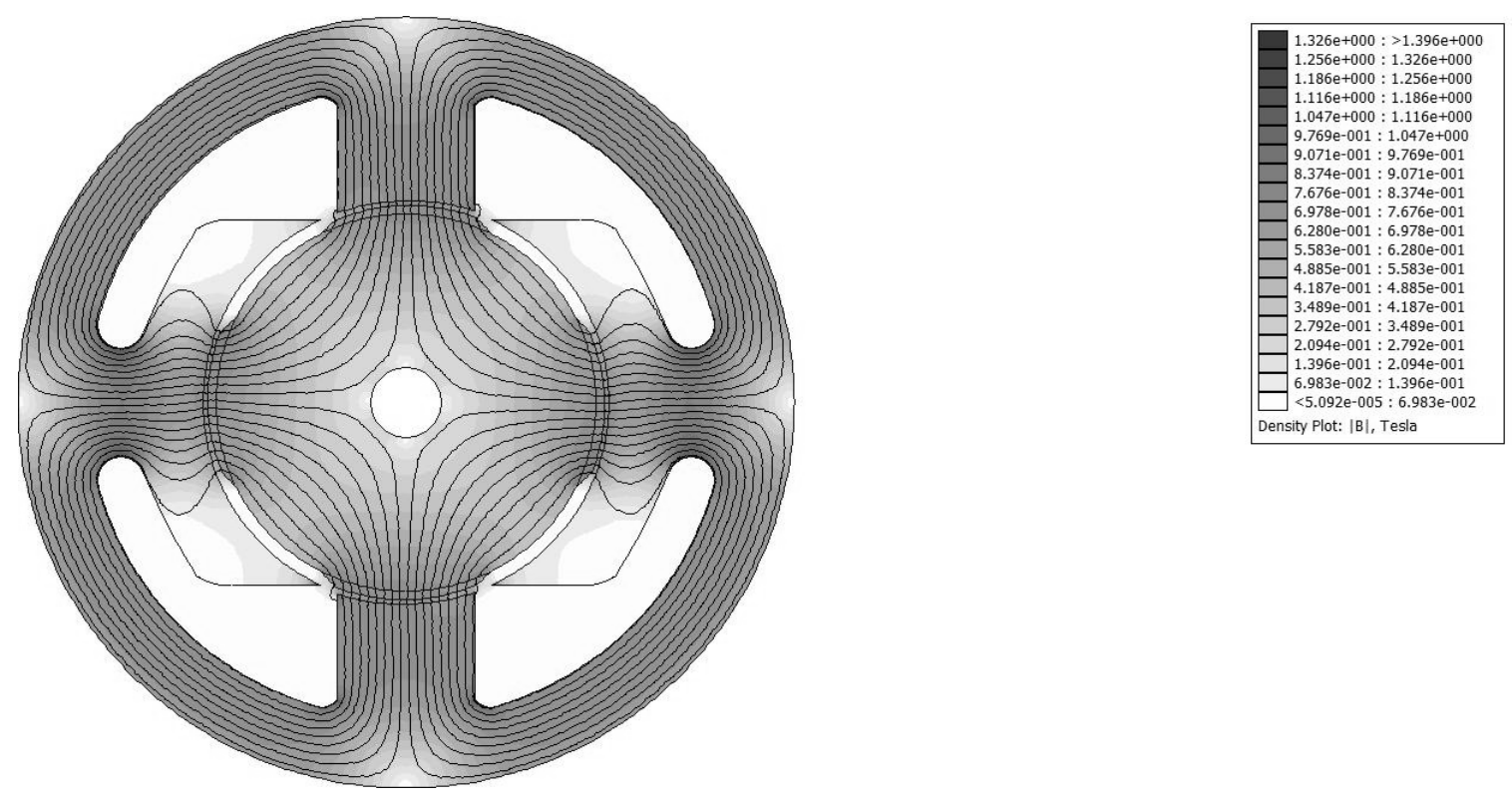

Fig. 9. Magnetic field distribution at no load for the PSO solution when the axes of the stator and rotor poles are aligned
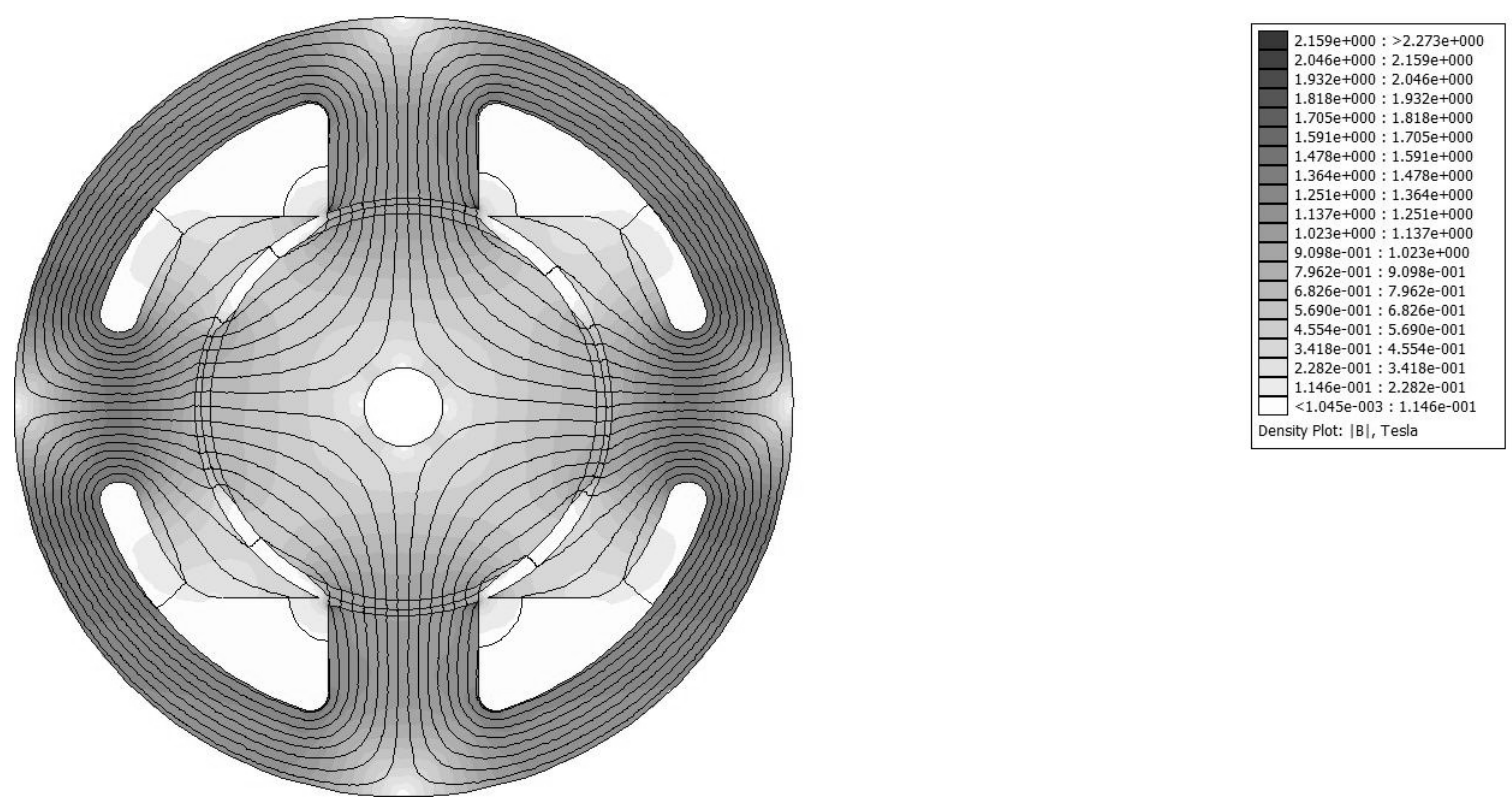

Fig. 10. Magnetic field distribution at rated load for the initial model when the axes of the stator and rotor poles are aligned 

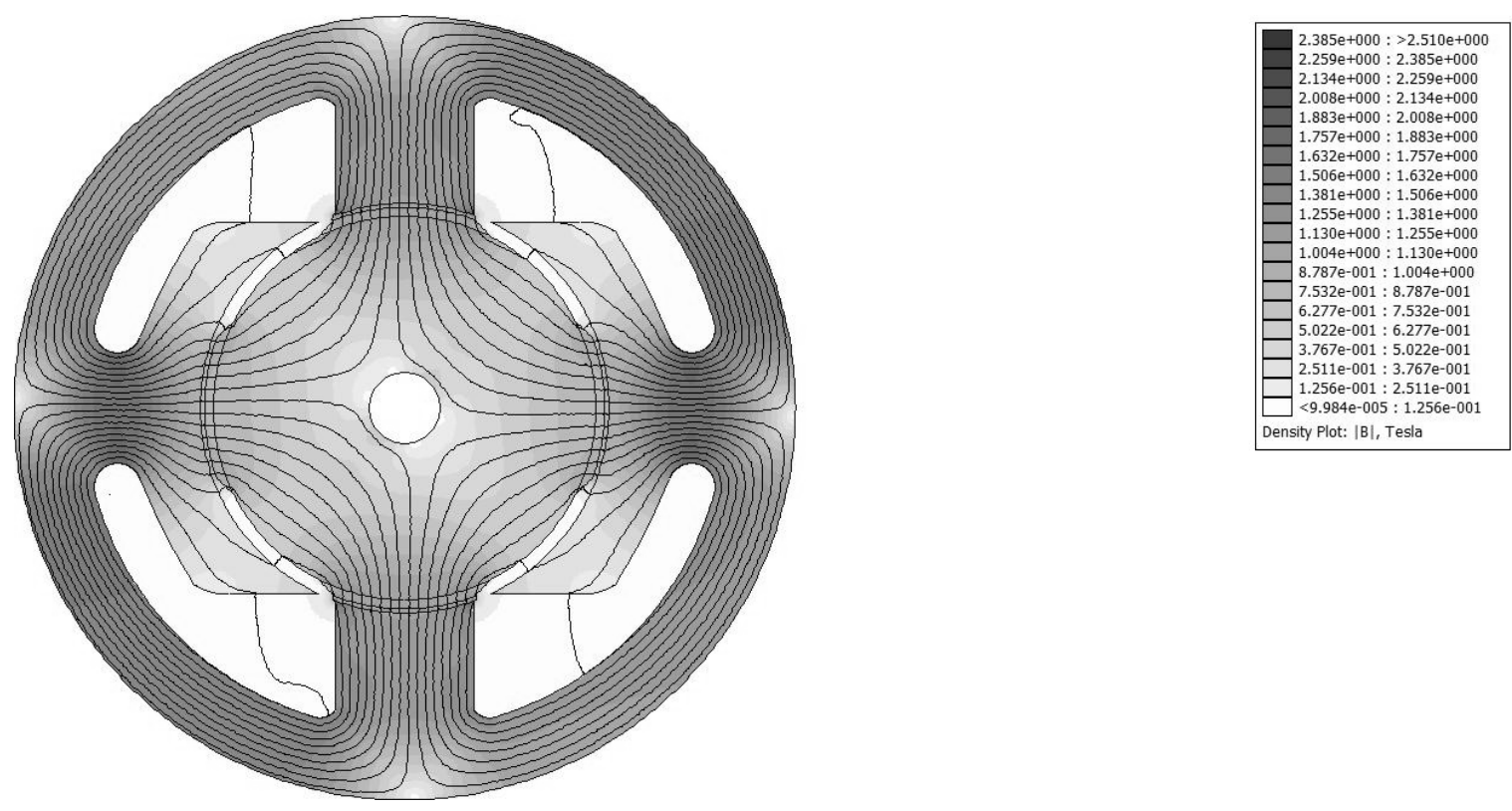

Fig. 11. Magnetic field distribution at rated load for the PSO solution when the axes of the stator and rotor poles are aligned

\section{Air gap Flux Density}

In the postprocessor mode of the program using the data from the magnetic field calculation, the value of the air gap flux density in the middle of the air gap can be calculated by using equation (2) and solving it numerically.

$\mathbf{B}=\operatorname{curl} \mathbf{A}$

(2)

The value of the air gap flux density for the single phase BLDC motor was calculated for different load currents. Due to limited space, the distribution of the air gap flux density is only presented for the SPBLDCM models at no load as shown in Fig. 12, and Fig. 13 , respectively.

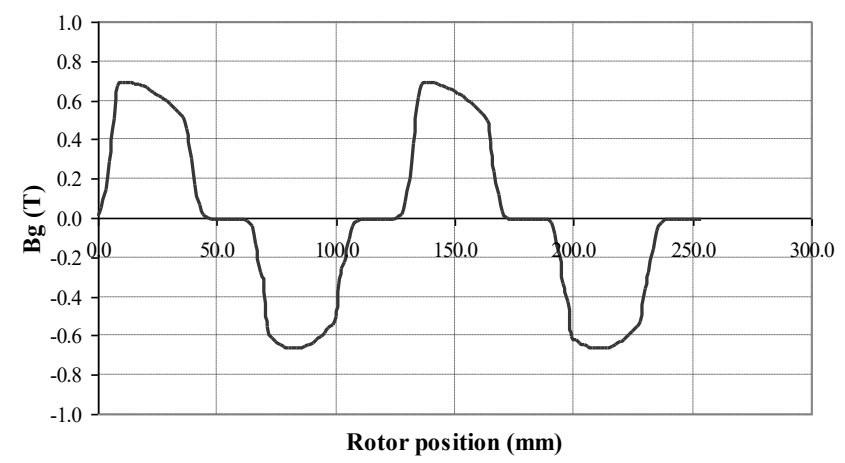

Fig. 12. Air gap flux density distribution at no load for the initial model

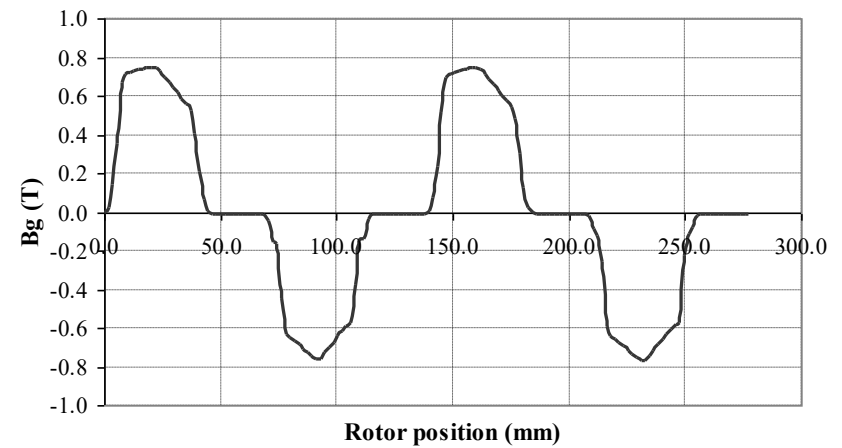

Fig. 13. Air gap flux density distribution at no load for the PSO solution 
In Fig. 14 and Fig. 15 a flux density distribution at rated load for an aligned stator and rotor poles is also presented. As a result of the change of the dimensions of the motor, the air gap flux density shape and magnitude has improved in the PSO solution in relation to the prototype model. How these changes will affect the overall performance of the motor will be analysed in some near future work.

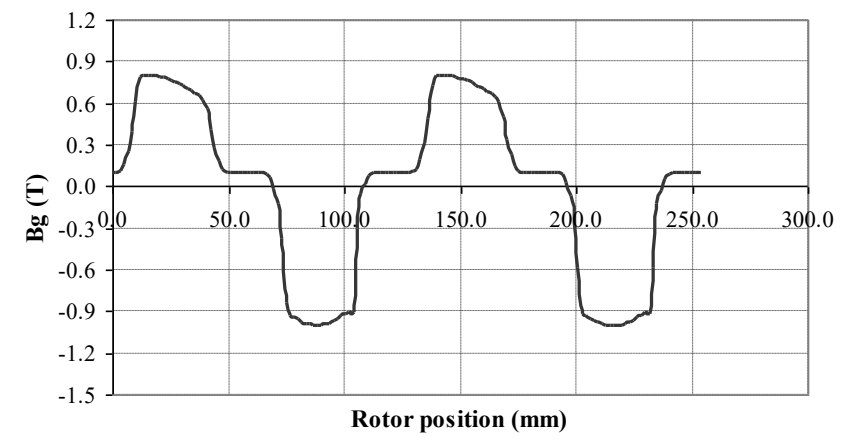

Fig. 14. Air gap flux density distribution at rated load for the initial model when the axes of the stator and rotor poles are aligned

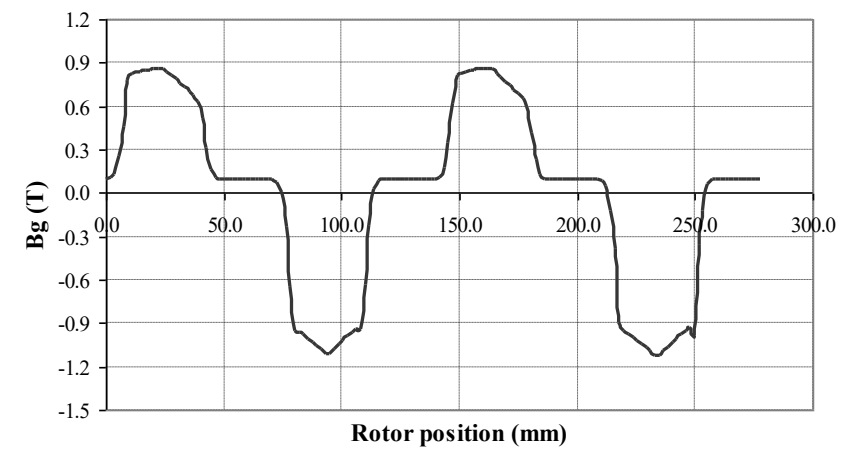

Fig. 15. Air gap flux density distribution at rated load for the PSO solution when the axes of the stator and rotor poles are aligned

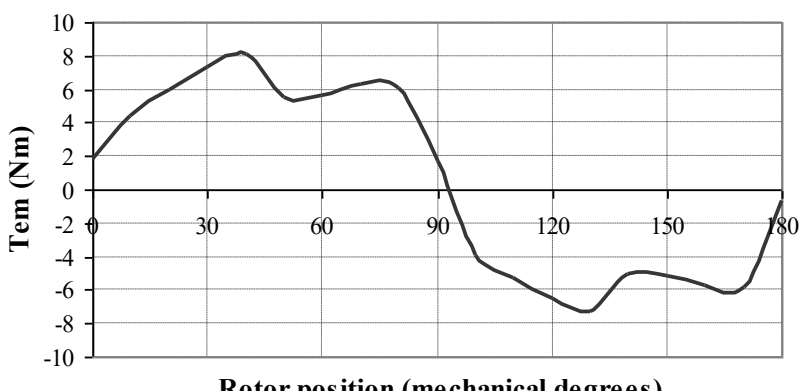

Rotor position (mechanical degrees)

Fig. 16. Electromagnetic torque spanned over a pair of poles (initial model)

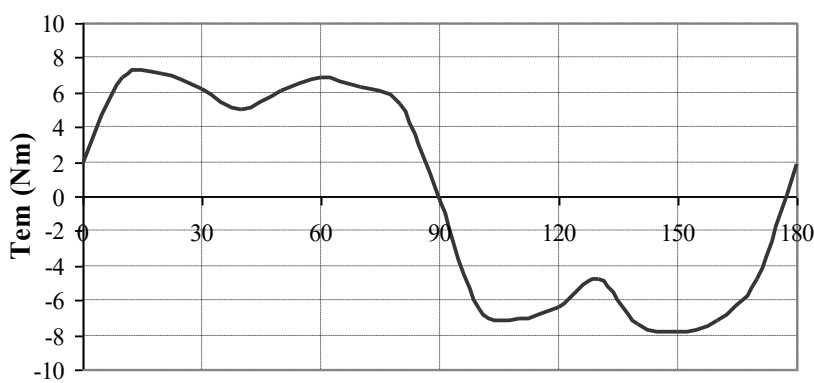

Rotor position (mechanical degrees)

Fig. 17. Electromagnetic torque spanned over a pair of poles (PSO model)

\section{Electromagnetic Torque}

The electromagnetic torque of the initial and PSO solution of the motor is solved in the FEM post processor using an additional Laplace equation, and the weighting function is computed. The stress tensor is then evaluated as volume integration, and the results are displayed. These results are typically more accurate than when a Maxwell Stress Tensor line integral is used, since all possible computational contours have been averaged to yield the torque result. The static electromagnetic torque of the initial motor is calculated at a rated current of $3.28 \mathrm{~A}$, and is presented in Fig. 16. Also the static electromagnetic torque of the PSO solution is 
calculated at a rated current of $3.5 \mathrm{~A}$, and is presented in Fig. 17. The torque characteristic is evaluated for one half of a rotation, i.e. $180^{\circ}$ mechanical or $360^{\circ}$ electrical.

\section{Conclusion}

An optimisation technique based on particle swarm optimisation has been developed and applied to the design of a SPBLDC motor. According to the results investigated above, it can be concluded that the PSO is a very suitable tool for design optimisation of SPBLDCM and electromagnetic devices in general. By using PSO the risk of trapping in a local maximum or minimum is extremely reduced, which is very difficult to eliminate in deterministic methods. At the end the quality of the PSO model has been proved through the data analysis of the prototype and PSO solution. This improvement resulted in energy efficiency improvement of the motor which is very important for the overall improvement of the motor performance. At the end, the quality of the PSO solution has been proved by comparative analysis of the two motor models using Finite Element Method as a performance analysis tool. The proper modelling of the SPBLDCM is presented and partial comparative results of the magnetic field and air gap flux density distribution for no load and rated load are presented. As an addition to the performance analysis of the motor the electromagnetic torque for both models is calculated and presented.

\section{References}

[1] Ahmed, S., and Lefley, P., (2009), "Development of a Single Phase PM BLDC Motor from a Novel Generic Model", Proceedings of the 11th Spanish Portuguese Conference on Electrical Engineering-11CHLIE, Zaragoza, Spain, pp. 1-6.

[2] Eberhart, R. C., Kennedy, J., (1995), "A new optimizer using particle swarm theory", Procedings of the 6th International Symposium Micromachine Human Science, Nagoya, Japan, pp. 39-43.

[3] Iepure, L., Tutelea, L., Boldea, I., (2008), "FEM analysis and control of a tapered air-gap single phase PMSM," Proceedings of the 11th International Conference on Optimization of Electrical and Electronic Equipment - OPTIM'2008, pp. 241-248.

[4] Kennedy, J. Eberhart, R.C., (1995), "Particle swarm optimization", Proceedings of IEEE International Conference on Neural Network, Perth, Australia, vol. 4, pp. 1942-1948.

[5] Kennedy, J. Eberhart, R. C. Shi, Y., (2001), Swarm Intelligence, The Morgan Kaufmann Series in Evolutionary Computation, Morgan Kaufmann Publishers, San Francisco.

[6] Lefley, P., Petkovska, L., Ahmed, S., Cvetkovski, G., (2010), "Finite Element Analysis of a Novel Single Phase Permanent Magnet Brushless DC Motor, Proceedings of the $14^{\text {th }}$ International Power Electronics and Motion Control Conference-EPE-PEMC 2010 on CD, Ohrid, Macedonia, pp.T4-96-101.

[7] Meeker, D., (2010), User's Manual for Finite Element Method Magnetics FEMM Software, ver. 4.2. 